\title{
Temporal sensitivity changes with extended training in a bisection task in a transgenic rat model
}

\author{
Bruce L. Brown 1,2, Sophie Höhn ${ }^{3,4}$, Alexis Faure ${ }^{3,4}$, Stephan von Hörsten ${ }^{5}$, Pascale Le Blanc ${ }^{3,4}$, \\ Nathalie Desvignes ${ }^{3,4}$, Nicole El Massioui ${ }^{3,4}$ and Valérie Doyère ${ }^{3,4}$ * \\ 1 Department of Psychology, Queens College, Flushing, NY, USA \\ 2 Department of Psychology, Graduate Center, City University of New York, New York, NY, USA \\ ${ }^{3}$ Centre de Neurosciences Paris-Sud, Université Paris-Sud, UMR 8195, Orsay, France \\ ${ }^{4}$ CNRS, Orsay, France \\ ${ }^{5}$ Experimental Therapy, Franz Penzoldt Center, Friedrich-Alexander University, Erlangen-Nürnberg, Germany
}

Edited by:

Warren H. Meck, Duke University, USA

\section{Reviewed by:}

Rosario Moratalla, Consejo Superior de Investigaciones Científicas, Spain Toshimichi Hata, Doshisha University, Japan

\section{${ }^{*}$ Correspondence:}

Valérie Doyère, Centre de

Neurosciences Paris-Sud, CNRS-UMR

8195, Université Paris-Sud, Bat. 446,

91405 Orsay, France.

e-mail: valerie.doyere@u-psud.fr
The present study investigated temporal perception in a Huntington disease transgenic rat model using a temporal bisection procedure. After initial discrimination training in which animals learned to press one lever after a 2-s tone duration, and the other lever after a 8-s tone duration for food reward, the bisection procedure was implemented in which intermediate durations with no available reinforcement were interspersed with trials with the anchor durations. Bisection tests were repeated in a longitudinal design from 4 to 8 months of age. The results showed that response latencies evolved from a monotonic step-function to an inverted U-shaped function with repeated testing, a precursor of non-responding on trials with intermediate durations. We inferred that temporal sensitivity and incentive motivation combined to control the transformation of the bisection task from a two-choice task at the outset of testing to a three-choice task with repeated testing. Changes in the structure of the task and/or continued training were accompanied by improvement in temporal sensitivity. In sum, the present data highlight the possible joint roles of temporal and nontemporal factors in the temporal bisection task, and suggested that non-temporal factors may compensate for deficits in temporal processing.

Keywords: temporal bisection, temporal discrimination, latency, sensitivity, non-sensory factors, transgenic rat model, Huntington disease

\section{INTRODUCTION}

The temporal bisection procedure has been used extensively to study temporal perception in animals. The procedure is a variant of the classical psychophysical method of constant stimuli. It entails an initial conditional discrimination training phase in which one response is rewarded following a short-duration stimulus, while the other response is rewarded following a long-duration stimulus. In a subsequent test phase, the short and long anchor stimuli are presented in addition to intermediate test durations, which are assumed to encompass an interval of uncertainty within which stimuli are indiscriminable from each other (Woodworth and Schlosberg, 1954). A virtue of the method is that it can provide separate measures of the point of subjective equality (PSE), difference limen and Weber fraction that are extracted from the resulting psychometric function. Variations in these measures are assumed to reflect the operations of fundamental mechanisms of temporal perception.

The original purpose of the current study was to investigate temporal perception in a Huntington disease (HD) transgenic rat model. In this model, the striatum is one of the primary structures affected, with the first motor symptoms appearing around 6 months of age and striatal neurodegeneration detectable from 8 months onward (von Horsten et al., 2003; Nguyen et al., 2006). As prefronto-striatal circuits are thought to play a critical role in temporal processing (Buhusi and Meck, 2005), we sought to track the concomitant deterioration in timing behavior over the course of 4-8 months in homozygous models and wt controls. While deficits in timing behavior have been observed at 4 months (Höhn et al., 2011), the data reported here suggest that non-temporal factors may counteract deficits in temporal processing.

\section{MATERIALS AND METHODS}

The procedure and apparatus have been reported elsewhere (see Höhn et al., 2011). Details specific to the present study are reported here.

\section{ANIMALS}

A cohort of nine wild type (wt) and 12 transgenic (tgHD) rat models for HD was imported at 3 months old (von Horsten et al., 2003). Subjects were housed in pairs in a temperature- and humiditycontrolled colony room $\left(23^{\circ} \mathrm{C}, 41 \%\right.$ humidity), with a light-dark cycle of 12:12 (lights on at 08:00 AM). After 2 weeks of adaptation, daily food rations were progressively reduced until rats reached $80 \%$ of their initial weight before the start of training, and the rats were maintained at $85 \%$ of normative weight afterward. All experiments were carried out in accordance with the recommendations of the EEC (86/609/EEC) and the French National Committee $(87 / 848)$ for care and use of laboratory animals. 


\section{APPARATUS}

Four operant Skinner boxes $(31 \mathrm{~cm} \mathrm{~W} \times 25 \mathrm{~cm} \mathrm{D} \times 31 \mathrm{~cm} \mathrm{H})$ in soundproof ventilated chambers (background noise $65 \mathrm{~dB}$ ) were controlled with the Graphic State program (Coulbourn Instruments, Harvard Apparatus, USA). On the left panel were a pellet dispenser for delivery of $45 \mathrm{mg}$ grain-based precision pellets in a food cup, and two $4-\mathrm{cm}$ retractable response levers. A speaker was located on the opposite side of the box and permitted delivery of an auditory stimulus $(1 \mathrm{kHz}, 80 \mathrm{~dB})$. At the beginning of each session, a red house light was illuminated.

\section{BEHAVIORAL PROCEDURES}

After an initial training phase for temporal discrimination, bisection tests were run monthly in a longitudinal design from 4 to 8 months. The temporal discrimination and the bisection test procedures were identical to those reported previously (Callu et al., 2009; Höhn et al., 2011). Animals were run in six cohorts of four transgenic and wt rats, and were fed in their home cage at the end of the experimental session.

\section{Pretraining}

At 3 months of age, rats were magazine trained in one session (30 pellets delivered on a variable time $60 \mathrm{~s}$ schedule). The next 2 days, they were trained on a continuous reinforcement schedule for each lever separately until 50 reinforcements were earned.

\section{Temporal discrimination training}

Reponses to one of two levers (left vs. right) were reinforced following one of two tone durations ( 2 vs. $8 \mathrm{~s}$ ). Two blocks of 40 trials, for a total of 80 trials, were presented with equal probability for each tone duration in each of 17 sessions. The relation of tone duration and reinforced response location was counterbalanced between groups. The levers were retracted immediately after a response or after $5 \mathrm{~s}$. The inter-trial interval (ITI) was $30 \mathrm{~s}$ on average (range $20-40 \mathrm{~s}$ ).

\section{Bisection tests}

Rats were then tested in a psychophysical choice procedure with five intermediate durations $(2.5,3.2,4,5$, and $6.3 \mathrm{~s})$ on nonreinforced trials ( 12 trials each duration), in addition to the two training anchor durations (2 and $8 \mathrm{~s}, 60$ trials each) with reinforcement available. The mean ITI was $30 \mathrm{~s}$. Four to six bisection sessions were run each month, from 4 to 8 months, followed by one session of discrimination training.

\section{DATA ANALYSIS}

Response location and latency were recorded for each trial. Analysis of latency included only trials with a response. Bisection data were calculated as proportion of responses on the lever assigned as correct for the long-duration stimulus on all trials with response. The bisection function relating proportion "long" responses to stimulus duration is typically sigmoidal in shape. The stimulus value corresponding to $p$ ("Long") $=0.5$ typically falls at the geometric mean of the anchor durations PSE. The slope of the function in the vicinity of the PSE reflects temporal sensitivity. The bisection function was analyzed with the pseudo-logistic model (PLM; Killeen et al., 1997) fit for each rat on the averaged curve obtained at each month, using Prism software; With the assumption that scalar variance dominated (Allan, 2002; Callu et al., 2009), the fits were good (median proportion of variance accounted for $=0.997$ and 0.995 for wt and tgHD groups, respectively). The PSE and the temporal sensitivity parameter (gamma) were estimated for each rat using the following formula (Allan, 2002, Eq. 5):

$P\left(R_{L}\right)=\left[1+\exp \left(\frac{T_{1 / 2}-t}{\frac{\sqrt{3}}{\pi} \gamma t}\right)\right]^{-1}$

Gamma, which is proportional to the Weber fraction, increases as temporal sensitivity decreases.

Contrast analyses of variance (ANOVAs; Rouanet et al., 1990) with an alpha level of 0.05 were used for statistical assessments.

\section{RESULTS}

\section{RESPONSE LATENCY AND PERCENT RESPONSE}

As reported elsewhere for these animals (Höhn et al., 2011), performances during initial temporal discrimination training were similar for wt and tgHD rats. At 4 months, tgHD rats showed a typical bisection curve, with similar PSE but poorer sensitivity (higher gamma) than wt. Both groups showed a decrease in response latency with increasing stimulus duration.

During the 25 bisection test sessions that were conducted over a 5-month period, both wt and tgHD rats progressively learned not to respond following intermediate durations, with no available reinforcement, while responding was maintained on trials with the anchor durations. The latter trend was evidenced in a sharp decrease in the mean percent of trials with a response (percent response) as intermediate values approached the 4-s geometric mean of the anchor duration values, and an increase in mean response latency which peaked near the geometric mean of the anchor durations (Figures 1A,B). On the final session, the minimum percent response on a given intermediate duration varied between 0 and $100 \%$ for $\operatorname{tgHD}$ rats, and between 16.67 and $100 \%$ for wt rats. Four of $12 \operatorname{tgHD}$ and five of nine wt rats still responded on at least $95 \%$ of trials on average for the intermediate durations. Performance was analyzed for these subgroups of animals who had stopped responding or not (with a criterion of 95\%) on the final session. On the 4-month test, in addition to the decrease in latency with stimulus duration previously reported (Höhn et al., 2011), an ANOVA indicated that mean latency for subgroup "stop" significantly exceeded that for subgroup "no-stop" [137 ms; $F(1,17)=6.43, p<0.05]$, with no effects involving genotype $(F s<1)$. In order to characterize the development of performance patterns that foreshadowed the cessation of responding, the difference in latency was calculated between the test session immediately prior to cessation of responding for each animal and the 4-month test (Figure 1C right panel). For the percent response measure (Figure 1C left panel), the difference calculation was based on the 4-month test and the last (25th) test session. Similar inverted U-shaped curves for latency and U-shaped curves for percent response were obtained for wt and tgHD rats 

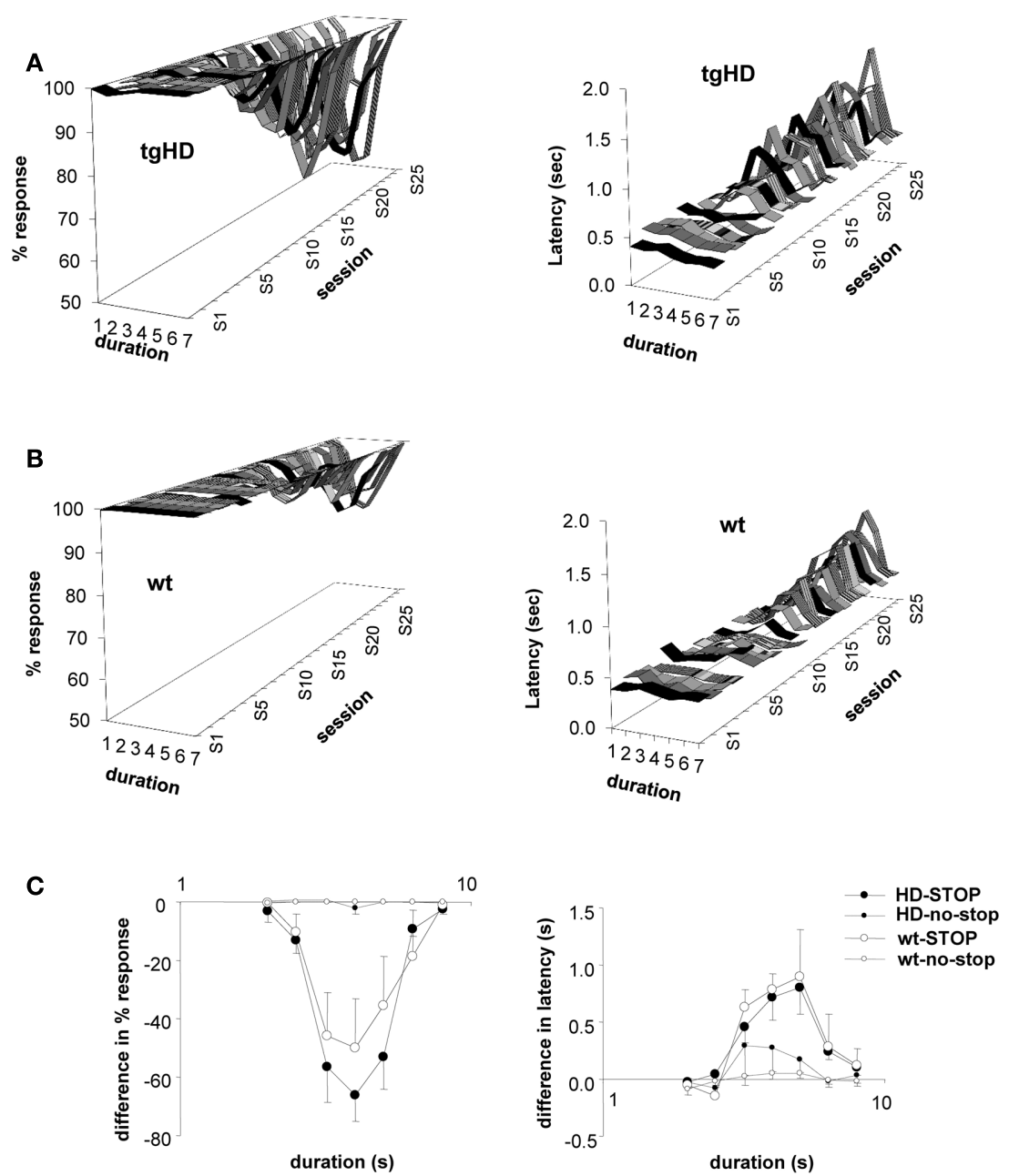

FIGURE 1 | Increased response latency for intermediate durations leads to cessation of responding. Percent responses (left) and response latencies (right) across signal durations and sessions during temporal bisection tests for tgHD (A) and wt (B) rats. In (C) left panel, mean ( \pm SEM) difference in percent responses between the last test session and the 4-months tests for subgroups of animals that stopped responding or not to

intermediate durations (with a criterion of $95 \%$ ). In (C) right panel, mean $( \pm$ SEM) difference in response latencies between the 4-months tests and (a) the last test session prior to stopping responding (95\% criterion) for subgroups of animals that stopped responding or (b) the last test session for the no-stop subgroups. For both panels, data are shown as a function of both genotype and duration.

which stopped responding. In genotype $\times$ subgroup $\times$ duration ANOVAs for both dependent measures, there were no significant effects involving genotype (all $F \mathrm{~s}<1$ ). Curves for the stop subgroup differed significantly from the curves of the no-stop subgroup [subgroup $\times$ duration interaction, $F(6,102)=4.63$ and $F(6,102)=14.21, p s<0.001$, for response latency and percent response, respectively]. While the stop and no-stop subgroups did not differ at the two anchor durations $[F(1,17)=1.59$ and $F<1$ for response latency and percent response, respectively], they differed significantly at the middle three intermediate durations $[F(1,17)=12.29$ and $F(1,17)=25.31 ; p<0.01$ for response latency and percent response]. Thus, the greater effect of duration on latency for the stop subgroup compared to the no-stop subgroup was associated with subsequently observed differences in the tendency to cease responding to non-reinforced intermediate durations.

\section{TEMPORAL SENSITIVITY AND PERCENT RESPONSE}

The foregoing findings represent the acquisition of a temporal discrimination between intermediate and anchor durations in the bisection protocol. One factor that may control the speed of acquisition of that discrimination is temporal sensitivity. Considering tgHD rats only, which showed a large variability between animals in temporal sensitivity at 4 months (Höhn et al., 2011), there was a significant positive correlation between gamma measured at 4 months and the session number at which the percent response measure fell below 95\% [Figure 2A, $r(10)=0.60$, $p<0.04]$. The significant correlation held when considering both wt and tgHD rats [ $z$-scores, $r(19)=0.50, p<0.03$ ]. Thus, better temporal sensitivity at 4 months predicted faster acquisition of the tendency to stop responding on intermediate durations. Owing to stable high levels of responding to the anchor durations throughout testing, this tendency reflected the acquisition 

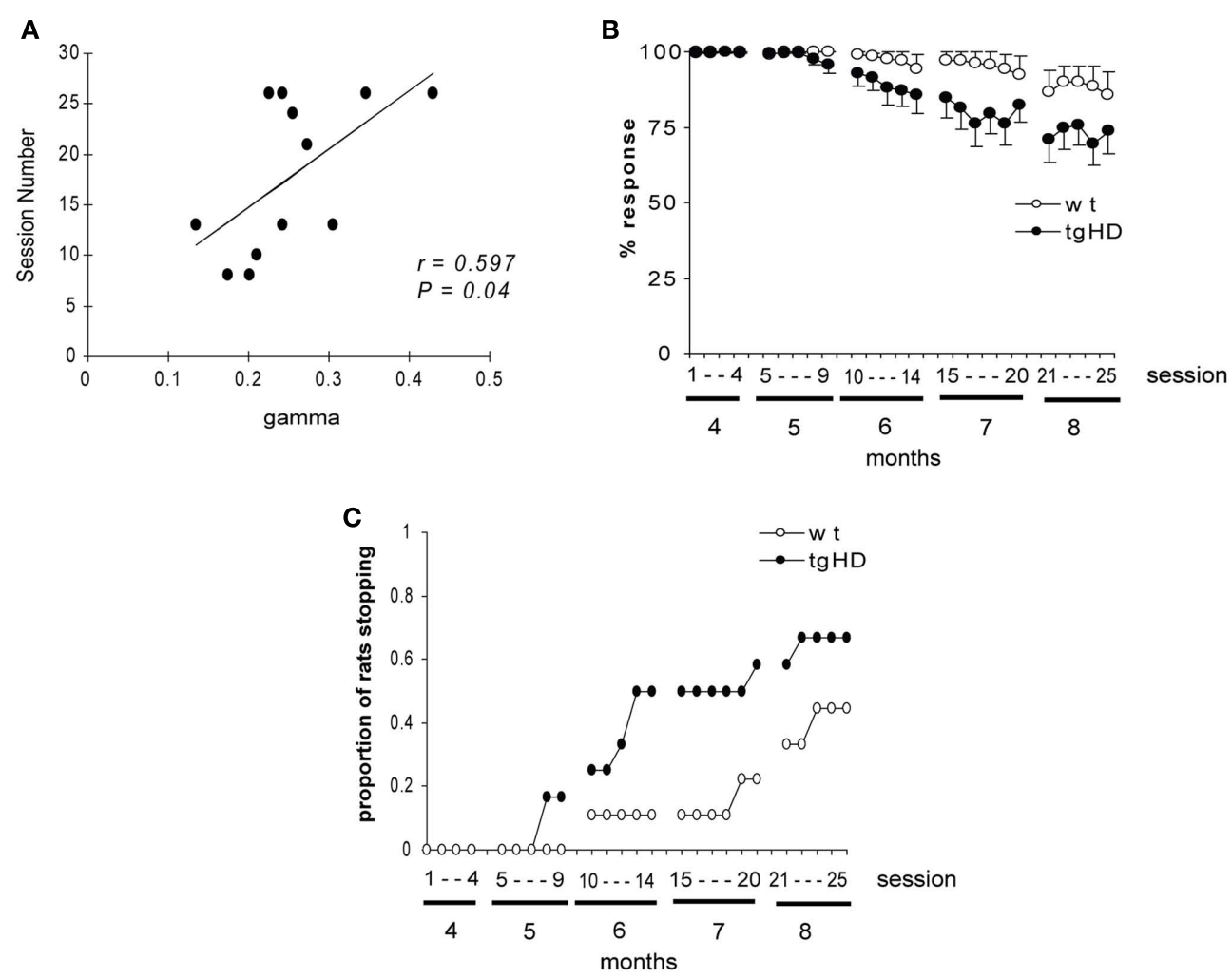

FIGURE 2 | During the course of bisection testing, tgHD animals stop responding to intermediate stimulus durations earlier than wt animals do. (A) Correlation between the session number at which the rats stop responding to intermediate durations (criterion < 95\%) and gamma measured at 4 months, for tgHD rats. (B) Mean ( \pm SEM) percent responses to intermediate durations on the 25 sessions of testing in months 4-8 for tgHD (filled symbols) and wt (empty symbols) rats. (C) Proportion of rats in each group stopping to respond across the 25 sessions of testing. of a discrimination between intermediate durations and anchor durations.

As mentioned above, $\operatorname{tgHD}$ rats showed on average poorer sensitivity at 4 months than wt, and therefore would be expected to be slower in learning the discrimination. The opposite was observed, however, as tgHD rats stopped responding to intermediate durations earlier than wt rats as shown in Figure 2B [significant group $\times$ session interaction, $F(24,456)=1.87, p<0.01]$. A trend toward faster learning was also observed when calculating the proportion of animals in each group that stopped responding, for each of the 25 test sessions (Figure 2C), although the difference in proportion observed during sessions 13 to 18 was only marginally significant (Fisher Exact Test, $p=0.07$ ). Thus, other factors in addition to temporal sensitivity combine to govern temporal performance in the bisection task.

\section{TEMPORAL BISECTION FUNCTION}

The fact that animals progressively stopped responding to intermediate durations precluded the analysis of the bisection curves for these animals with repeated testing. Fewer than 50 percent of the total number of animals (four tgHD and five wt) were responding at 8 months, whereas more than $50 \%$ were still responding at 7 months. When restricting the analysis to animals that were responding at the 7 -month testing phase (wt, $n=7 ; \operatorname{tg} \mathrm{HD}, n=6)$, the analysis of this subset confirmed that at 4 months of age tgHD rats showed poorer sensitivity, but similar PSE, compared to wt rats [Figures 3A,B, $F(1,11)=8.12$, $p<0.02$, and $F<1$, for gamma and PSE, respectively]. Interestingly, both PSE and gamma decreased with repeated testing across the 4 months $[F(3,33)=3.38, p<0.03, F(3,33)=8.39, p<0.001$, respectively]. Furthermore, while PSE did not change differentially between genotypes (no group $\times$ test month interaction, $F<1$ ), gamma tended to decrease more rapidly in tgHD than in wt rats. Both groups showed a significant decrease in gamma across months $[F(3,18)=3.80$ and $F(3,15)=5.61 ; p s<0.05$, for wt and tgHD groups respectively]. However, a marginally significant group $\times$ test month interaction $[F(3,33)=2.80, p=0.055]$, in addition to the fact that gamma for $\operatorname{tgHD}$ was significantly lower than for wt at 7 months of age $[F(1,11)=6.28, p<0.03]$ show that temporal sensitivity improved faster for the tgHD than for wt rats. These data indicate that PSE and gamma were differentially sensitive to repeated testing.

\section{DISCUSSION \\ INCREASED RESPONSE LATENCY FOR INTERMEDIATE DURATIONS LEADS TO CESSATION OF RESPONDING}

On initial test sessions, both groups showed a decrease in response latency with increasing test stimulus duration (Höhn et al., 2011), 

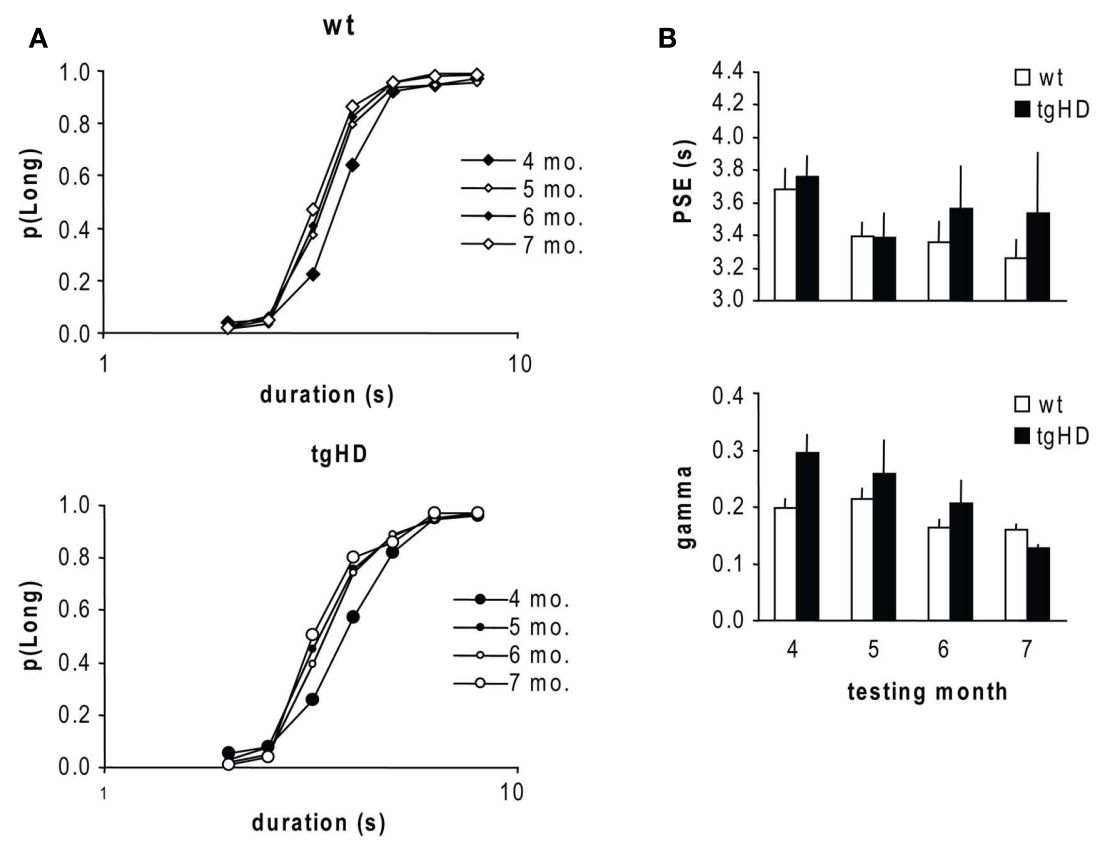

FIGURE 3 | Repetition of bisection testing sharpens temporal sensitivity. (A) Evolution of bisection curves for test months 4-7 for subgroups of animals that were responding at 7 months (top, wt rats; bottom, tgHD rats). (B) Mean (+SEM) PSE (top) and gamma (bottom) values estimated from the bisection curves for test months 4-7 represented in (A). replicating previously reported findings (Callu et al., 2009). The present data demonstrate that in rats, an inverted U-shaped function of response latency emerges as the animals learn to discriminate the intermediate durations from the anchors, and that it leads to cessation of responding to intermediate durations with continued testing. The inverted U-shaped function has been reported previously with both rat and human subjects (Maricq and Church, 1983; Meck, 1983; Rodríguez-Gironés and Kacelnik, 1998). Maricq and Church suggested that elevated latencies for intermediate durations in rats reflect either response conflict or discrimination learning between anchor vs. intermediate durations signaling availability vs. non-availability of reinforcement, respectively. The present data support the latter view, as the inverted U-shaped function was absent during initial test sessions, and was followed by the selective reduction in probability of responding to the intermediate test durations. Thus, intermediate durations used in the present study were discriminatively different from the anchors as indicated by performance in well-trained animals, suggesting that the bisection task functionally shifted from a two- to a three-alternative (two anchors and intermediate durations) discrimination task with repeated testing.

\section{REPETITION OF BISECTION TESTING SHARPENS TEMPORAL SENSITIVITY}

Point of subjective equality decreased and temporal sensitivity increased with repeated testing in the present study. The changes in both variables may be unrelated, but a decision-theoretic account, the PLM (Killeen et al., 1997), predicts a decrease in the PSE, from the arithmetic mean of the anchor durations to the harmonic mean as a limit, as gamma decreases. The change in PSE was also accompanied by the emergence of a behavioral discrimination between anchor and intermediate stimulus durations. It is not clear how that discrimination is related to the assumptions of PLM, but it is possible that judgments of "short" vs. "not short" (intermediate and long) that emerge with training may determine the location of the criterion in that model, or response bias in a scalar expectancy theory (SET) account (Gibbon, 1981; Allan and Gibbon, 1991) of the indifference point in the bisection task.

The improvement in temporal sensitivity could also be related to the change in the functional properties of the discrimination task. The introduction of new, intermediate durations in the test phase represents an increase in discrimination difficulty, which has been shown to decrease Weber fraction in humans (see Ferrara et al., 1997). Alternatively, temporal sensitivity may increase as a result of repeated exposure to the test stimuli in the absence of a change in task structure, consistent with the sharpening of stimulus generalization gradients with pre-exposure to the training stimulus (Honey, 1990) or increased amounts of training (Brown, 1970).

The foregoing phenomena may be taken to represent the acquisition of stimulus control, that is, the establishment of behavioral control by specific differences in the properties of stimuli along one or more continua. Thus, animals may learn to identify relevant stimulus dimensions, and change their behavior accordingly, only with repeated discrimination training. Alternatively, stimulus control may be established immediately upon the initial exposure to the discriminative stimuli, with increased training serving only to bring behavior under sharper control by the prevailing contingencies of reinforcement. Thus, sharpening of psychometric 
functions in the present study may reflect the effect of repeated exposure to the contingencies of reinforcement on performance, while stimulus differences are fully discriminated early in training (Balsam et al., 2002; Drew et al., 2005). In support of this idea are the results of Droit-Volet and Izaute (2009) who manipulated the availability of a third "I do not know" response in the temporal bisection procedure in human observers. Adults who were given that response option exhibited sharper bisection functions (smaller Weber fractions) immediately in a single test session compared to control subjects with only the two standard response options. Thus, temporal sensitivity may depend upon available response options in both rats and humans, with difference in rate of emergence reflecting the use of verbal vs. contingency-based instructions.

\section{TEMPORAL AND NON-TEMPORAL FACTORS GOVERN THE TEMPORAL PERFORMANCE}

Initial measures of sensitivity to temporal properties of stimuli between $\operatorname{tgHD}$ and wt rats predicted a difference in speed of acquisition of a temporal discrimination that was opposite to the one observed, that is, at 4 months, the tgHD rats had poorer sensitivity (higher gamma) than wt, but the tgHD rats stopped responding to the intermediate duration sooner than wt. Thus, it is likely that other factors in addition to temporal sensitivity combine to govern temporal performance in the bisection task. Superior learning capacities in tgHD rats could be one factor, as increased prefronto-striatal plasticity has been reported in presymptomatic tgHD rats (Höhn et al., 2011). However, initial learning of the simple 2 vs. $8 \mathrm{~s}$ discrimination was similar for tgHD and wt rats, although a more challenging discrimination task might have revealed a difference in temporal learning. In addition, Höhn et al. (2011) reported that at 10 months, the same tgHD rats were inferior to wt in learning a discrimination reversal. Therefore, superior learning is not a likely factor, and increased plasticity in young adult tgHD rats may be an index of compensatory mechanisms secondary to dysfunctional networks. Another factor may reside in age-related neurodegeneration in transgenic animals. The monotonic continuous decrease in percent response observed across sessions (Figure 2B), however, more likely reflects learning through repetition rather than an age-related effect (which would be expected to produce decreases between but not within months). Yet another factor may be greater sensitivity to nonreinforcement in tgHD rats, as responses to intermediate durations were never reinforced. In a separate study (Faure et al., 2011), using a runway task in which the sucrose reinforcement was suddenly changed to a lower or a higher concentration (i.e., a less or more rewarding value) tgHD rats were more reactive than wt rats to changes in reward values. It is therefore likely that incentive motivation is a factor in the speed of discrimination learning between anchor (reinforcement) and intermediate (no reinforcement) durations.

\section{CONCLUSION}

Our data highlight the dynamic properties of the temporal bisection procedure. A number of factors have been shown to modulate performance in that procedure in animals and humans, including pharmacological agents (e.g., Meck, 1983; Santi et al., 2001), temporal and non-temporal stimulus properties (e.g., Church and Deluty, 1977; Penney et al., 2000), arousal/emotion (e.g., Droit-Volet and Wearden, 2002; Grommet et al., 2011), lesion (e.g., Meck et al., 1984; Breukelaar and Dalrymple-Alford, 1999), and disease (e.g., Smith et al., 2007; Carroll et al., 2008). The present study shows that repetition or amount of training/testing is another relevant variable (see also Machado and Keen, 2003). Repetition resulted in a sharpening of the bisection function and discrimination between anchor durations and intermediate test durations, both of which were more pronounced in tgHD rats. This difference was correlated with responsiveness to motivational factors, reflecting higher sensitivity to changes in reward values.

Transgenic Huntington disease animals exhibit poorer temporal sensitivity at 4 months than wt animals, as shown in two different ways in Höhn et al. (2011). We show here that higher temporal sensitivity was correlated with earlier stopping to respond to intermediate non-reinforced durations during the course of bisection testing. The fact that tgHD animals stopped earlier than wt animals, in contrast to what would be expected from their poorer initial temporal sensitivity, shows that the speed with which stopping to respond occurs does not reflect only temporal sensitivity. We hypothesize that sensory factors and non-sensory (motivational) factors may play competing roles in temporal bisection performance, and that an enhanced sensitivity to non-reinforcement can offset lower levels of temporal sensitivity to produce more rapid expression of the discrimination between anchor vs. intermediate stimulus durations. While initial bisection testing provides a valid measure of temporal sensitivity (gamma), repeated testing in the bisection task provides an opportunity for motivational factors (reinforcement vs. nonreinforcement) to exert their effect on the tendency to respond vs. not respond.

Changes in temporal performance after genetic or other biological manipulations may be related to non-sensory (motivational) factors in addition to sensory (temporal) factors, and control of these factors may be critical in determining underlying mechanisms. Inasmuch as the role of motivational factors in timing performance has been inferred in different timing protocols including both the peak interval procedure (e.g., Ward et al., 2009) and the bisection procedure in this report, the isolation of temporal control of behavior may require more sophisticated measures in the general case. A technical solution to the presumed motivational confound encountered in the present study would be to use narrow anchor duration ranges that closely encompass the interval of uncertainty, as assumed in the method of constant stimuli, thus preserving response tendencies along all test durations. Owing to the joint effects of both anchor stimulus range and repeated stimulus exposure, assessment of temporal sensitivity may require continued adjustments in stimulus range until stable performance is observed.

Sharpening of the bisection function with repetition may reflect an increase in temporal sensitivity apart from the influence of nontemporal factors. At an empirical level it is not known whether repeated exposure to the reinforcement contingencies involving the anchor durations or repeated exposure to the test durations was critical, as these variables were confounded in the present 
study. From the perspective of temporal information processing theory (SET, Gibbon et al., 1984), variation in temporal sensitivity could be related to variability in the perception of the anchor stimuli or in memory representation (Allan and Gerhardt, 2001), or possibly in the decision mechanism (Penney et al., 2008). A challenge for future research is the dissociation among these mechanisms as accounts of the effects of repeated training/testing upon performance in the bisection task.

\section{REFERENCES}

Allan, L. G. (2002). The location and interpretation of the bisection point. Q. J. Exp. Psychol. 55B, 43-60.

Allan, L. G., and Gerhardt, K. (2001). Temporal bisection with trial referents. Percept. Psychophys. 63, 524-540.

Allan, L. G., and Gibbon, J. (1991). Human bisection at the geometric mean. Learn. Motiv. 22, 39-58.

Balsam, P. D., Drew, M. R., and Yang, C. (2002). Timing at the start of associative learning. Learn. Motiv. 33, 141-155.

Breukelaar, J. W., and DalrympleAlford, J. C. (1999). Effects of lesions to the cerebellar vermis and hemispheres on timing and counting in rats. Behav. Neurosci. 113, 78-90.

Brown, B. L. (1970). Stimulus generalization in salivary conditioning. $J$. Comp. Physiol. Psychol. 71, 467-477.

Buhusi, C. V., and Meck, W. H. (2005). What make us tick? Functional and neural mechanisms of interval timing. Nat. Rev. Neurosci. 6, 755-765.

Callu, D., El Massioui, N., Dutrieux, G., Brown, B. L., and Doyère, V. (2009). Cognitive processing impairments in a supra-second temporal discrimination task in rats with cerebellar lesion. Neurobiol. Learn. Mem. 91, 250-259.

Carroll, C. A., Boggs, J., O’Donnell, B. F., Shekhar, A., and Hetrick, W. P. (2008). Temporal processing dysfunction in schizophrenia. Brain Cogn. 67, 150-161.

Church, R. M., and Deluty, M. Z. (1977). Bisection of temporal intervals. J. Exp. Psychol. Anim. Behav. Process. 3 , 216-228.

Drew, M. R., Zupan, B., Cooke, A., Couvillon, P. A., and Balsam, P. D. (2005). Temporal control of conditioned responding in goldfish. J. Exp. Psychol. Anim. Behav. Process. 31, 31-39.

Droit-Volet, S., and Izaute, M. (2009). Improving time discrimination in children and adults in a temporal bisection task: The effects of feedback and no forced choice on decision and memory processes. Q. J. Exp. Psychol. 62, 1173-1188.
Droit-Volet, S., and Wearden, J. (2002). Speeding up an internal clock in children? Effects of visual flicker on subjective duration. Q. J. Exp. Psychol. B. 55, 193-211.

Faure, A., Höhn, S., von Hörsten, S., Delatour, B., Raber, K., Leblanc, P., Desvignes, N., Doyère, V., and $\mathrm{El}$ Massioui, N. (2011). Altered emotional and motivational processing in the transgenic rat model for Huntington's disease. Neurobiol. Learn. Mem. 95, 92-101.

Ferrara, A., Lejeune, H., and Wearden, J. H. (1997). Changing sensitivity to duration in human scalar timing: an experiment, a review, and some possible explanations. Q. J. Exp. Psychol. B 50B, 217-237. [See Wearden and Ferrara (1995). QJEP. 49, 24-44].

Gibbon, J. (1981). On the form and location of the psychometric bisection function for time. J. Math. Psychol. 24, 58-87.

Gibbon, J., Church, R. M., and Meck, W. H. (1984). Scalar timing in memory. Ann. N. Y. Acad. Sci. 423, 52-77.

Grommet, E. K., Droit-Volet, S., Gil, S., Hemmes, N. S., Baker, A. H., and Brown, B. L. (2011). Time estimation of fear cues in human observers. Behav. Processes 86, 88-93.

Höhn, S., Dallérac, G., Faure, A., Urbach, Y., Nguyen, H. P., Riess, O., von Hörsten, S., Le Blanc, P., Desvignes, N., El Massioui, N., Brown, B. L., and Doyère, V. (2011). Behavevidence for presymptomatic alteration of prefronto-striatal processing in the transgenic rat model for Huntington disease. J. Neurosci. 31, 8986-8997.

Honey, R. C. (1990). Stimulus generalization as a function of stimulus novelty and familiarity in rats. J. Exp. Psychol. Anim. Behav. Process. 16, 178-184.

Killeen, P. R., Fetterman, J. G., and Bizo, L. A. (1997). "Time's causes," in Time and Behaviour: Psychological and Neurobehavioral Analyses, eds C. M. Bradshaw and E. Szabadi (Amsterdam: Elsevier), 79-131.

Machado, A., and Keen, R. (2003). Temporal discrimination in a long ioral and in vivo electrophysiological

\section{ACKNOWLEDGMENTS}

This work was supported by grants from Agence Nationale de la Recherche (ANR) and a Partner University Fund grant awarded to Valérie Doyère, and by a PSC-CUNY grant awarded to Bruce L. Brown by the Research Foundation of CUNY (Grant 61336-00 39). Sophie Höhn was supported by Ministry of Research (MRT). The publication of this paper is sponsored by the COST Action TIMELY.

operant chamber. Behav. Process. 62, 157-182.

Maricq, A. V., and Church, R. M. (1983). The differential effects of haloperidol and methamphetamine on time estimation in the rat. Psychopharmacology (Berl.) 79, 10-15.

Meck, W. H., Church, R. M., and Olton, D. S. (1984). Hippocampus, time, and memory. Behav. Neurosci. 98, 3-22.

Meck, WH. (1983). Selective adjustment of the speed of internal clock and memory processes. J. Exp. Psychol. Anim. Behav. Process. 9, 171-201.

Nguyen, H. P., Kobbe, P., Rahne, H., Wörpel, T., Jäger, B., Stephan, M., Pabst, R., Holzmann, C., Riess, O., Korr, H., Kántor, O., PetraschParwez, E., Wetzel, R., Osmand, A., and Von Hörsten, S. (2006). Behavioral abnormalities precede neuropathological markers in rats transgenic for Huntington's disease. Hum. Mol. Genet. 15, 3177-3194.

Penney, T., Gibbon, J., and Meck, W. (2000). Differential effects of auditory and visual signals on clock speed and temporal memory. J. Exp. Psychol. Hum. Percept. Perform. 26, 1770-1787.

Penney, T. B., Gibbon, J., and Meck, W. H. (2008). Categorical scaling of duration bisection in pigeons (Columba livia), mice (Mus musculus), and humans (Homo sapiens). Psychol. Sci. 19, 1103-1109.

Rodríguez-Gironés, M. A., and Kacelnik, A. (1998). "Response latencies in temporal bisection: implications for timing models," in Time and the Dynamic Control of Behavior, eds V. De Keyser, G. d'Ydewalle, and A. Vandierendonck (Seattle: Hogrefe \& Huber), 51-70.

Rouanet, H., Bernard, J.-M., and Le Roux, B. (1990). Analyse Inductive des Données: Statistique en Sciences Humaines. Paris: Dunod.

Santi, A., Coppa, R., and Ross, L. (2001). Effects of the dopamine D2 agonist, quinpirole, on time and number processing in rats. Pharmacol. Biochem. Behav. 68, 147-155.

Smith, J. G., Harper, D. N., Gittings, D., and Abernethy, D. (2007). The effect of Parkinson's disease on time estimation as a function of stimulus duration range and modality. Brain Cogn. 64, 130-143.

von Horsten, S., Schmitt, I., Nguyen, H. P., Holzmann, C., Schmidt, T., Walther, T., Bader, M., Pabst, R., Kobbe, P., Krotova, J., Stiller, D., Kask, A., Vaarmann, A., RathkeHartlieb, S., Schulz, J. B., Grasshoff, U., Bauer, I., Vieira-Saecker, A. M., Paul, M., Jones, L., Lindenberg, K. S., Landwehrmeyer, B., Bauer, A., Li, X. J., and Riess, O. (2003). Transgenic rat model of Huntington's disease. Hum. Mol. Genet. 12, 617-624.

Ward, R. D., Kellendonk, C., Simpson, E. H., Lipatova, O., Drew, M. R., Fairhurst, S., Kandel, E. R., and Balsam, P. D. (2009). Impaired timing precision produced by striatal D2 receptor overexpression is mediated by cognitive and motivational deficits. Behav. Neurosci. 123, 720-730.

Woodworth, R. S., and Schlosberg, H. (1954). Experimental Psychology. New York: Holt.

Conflict of Interest Statement: The authors declare that the research was conducted in the absence of any commercial or financial relationships that could be construed as a potential conflict of interest.

Received: 13 June 2011; paper pending published: 14 July 2011; accepted: 09 August 2011; published online: 28 September 2011.

Citation: Brown BL, Höhn S, Faure A, von Hörsten S, Le Blanc P, Desvignes $N$, El Massioui N and Doyère V (2011) Temporal sensitivity changes with extended training in a bisection task in a transgenic rat model. Front. Integr. Neurosci. 5:44. doi: 10.3389/fnint.2011.00044 Copyright (c) 2011 Brown, Höhn, Faure, von Hörsten, Le Blanc, Desvignes, El Massioui and Doyère. This is an openaccess article subject to a non-exclusive license between the authors and Frontiers Media SA, which permits use, distribution and reproduction in other forums, provided the original authors and source are credited and other Frontiers conditions are complied with. 